\title{
Sobre las fracciones continuas: aplicaciones y curiosidades
}

\author{
Manuel Murillo Tsijli \\ mmurillo@itcr.ac.cr \\ Escuela de Matemática \\ Instituto Tecnológico de Costa Rica \\ Recibido: Julio 14, $2014 \quad$ Aceptado: Diciembre 18, 2014
}

Resumen. En este artículo se hará una breve introducción al tema de las fracciones continuas. Como parte de sus aplicaciones, en primer lugar se muestra su utilidad para determinar la solución de una ecuación diofántica; en segundo, para obtener algunos criterios de divisibilidad; finalmente, se hace una breve explicación de cómo fueron utilizadas a la hora de corregir el calendario para obtener el que hoy se conoce como calendario gregoriano.

Palabras clave: Fracciones continuas, ecuación diofántica, divisibilidad, calendarios.

\begin{abstract}
This article presents a brief introduction to the subject of continued fractions. As part of their applications it is shown its utility for determining the solution of a diophantine equation and also, its use to obtain some divisibility criteria. Finally, we will to give a brief explanation about of how they were used on the correction of the calendar, until to get the current one, which it is known as the Gregorian calendar. Finally, a brief explanation is made on how they were used to correct the calendar in order to have what is known today as the gregorian calendar.
\end{abstract}

KeyWords: Continued fractions, diophantine equation, divisibility, calendars.

\subsection{Introducción}

Dos de las áreas de la matemática que, históricamente, se desarrollan primero son la aritmética y la geometría, es a partir de la primera de ellas que nace el álgebra. En general, se puede decir que la teoría de números estudia los números enteros y sus propiedades. Se desarrolló desde la antigüedad 
bajo el nombre de aritmética, del griego $\alpha \rho \imath \beta \mu$ ó (arithmos), número. Por un lado, tiene un atractivo relacionado con la solución de acertijos numéricos y problemas, y esto hace que los aficionados se recreen con esta teoría. Por otro lado, la dificultad derivada de la restricción propia de los enteros hace que muchos matemáticos se ocupen en la investigación de este campo.

El origen de la teoría de números se atribuye a los griegos, pues son los que dan la primera orientación científica al estudio de los enteros, más allá de la simple aritmética.

Pitágoras (572 - 497 a.C.), quien nació en la isla de Samos, Grecia, fue para algunos el primer matemático puro y se cree que fue discípulo de Tales. Es en esta isla donde fundó su primera escuela; luego, huyendo de la tiranía de Polícrates, estableció la segunda escuela en Crotona y la tercera en Tarento. Pitágoras y sus discípulos efectuaron un estudio bastante completo de los enteros, pues la filosofía de los pitagóricos se basaba en estos y los consideraban pilares del conocimiento, ellos creían que todas las cosas son, en esencia, números. Esta escuela aceptaba solamente los números enteros; para ellos, las fracciones no eran números.

Después de Euclides, no se efectuaron avances significativos en teoría de números hasta aproximadamente el 250 d.C., cuando otro matemático griego llamado Diofanto de Alejandría publicó su obra más importante, Arithmetica, compuesta por 13 libros, de los cuales se conservan solamente 6; esta es la primera obra griega en la que se realiza un uso sistemático de los símbolos algebraicos. Muchos de los problemas se originaron en la teoría de números y a él le pareció natural buscar soluciones enteras para las ecuaciones. Las que deben ser resueltas por medio de valores enteros de las incógnitas hoy se llaman ecuaciones diofánticas, y el estudio de tales ecuaciones recibe el nombre de análisis diofántico. Diofanto fue hábil para resolver ecuaciones algebraicas con dos o tres incógnitas, a pesar de que la notación que empleaba era un tanto incómoda. Tras Diofanto no se realizaron muchos progresos en teoría de números hasta el siglo XVII, si bien existe evidencia de que el tema empezó a crecer en el Lejano Oriente, especialmente en la India, en el periodo comprendido entre el 500 y el 1200 d.C. [9]. Por ejemplo Aryabhata (476-550) utilizó las fracciones continuas tanto para resolver ecuaciones diofánticas, como para dar aproximaciones a ciertos números irracionales. Por otra parte, Brahmagupta (598-668) profundizó en el estudio de las ecuaciones diofánticas de la forma $x^{2}-n y^{2}=1$ con $n \in \mathbb{Z}$, conocidas hoy como ecuaciones de Pell. Particularmente investigó las soluciones de la ecuación $x^{2}-61 y^{2}=1$ y encontró la menor solución: $(1766319049,226153980)$, para ello utilizó cálculos semejantes a los de las fracciones continuas.

En Italia, Rafael Bombelli (1526-1572) utilizó un precursor de las fracciones continuas para calcular aproximaciones de $\sqrt{13}$. Antonio Cataldi (1548-1626) se dio cuenta de que el método de Bombelli era válido para todas las raíces cuadradas y lo utilizó para calcular $\sqrt{18}$, además publicó que las aproximaciones obtenidas son alternadamente mayores y menores a la raíz buscada (corolario 1.2).

Posteriormente, en Inglaterra hubo avances significativos, William Brouncker (1620-1684) utilizó estas fracciones para construir una sucesión que convergía a $4 / \pi$, además, aproximó el valor de $\pi$ con 10 decimales significativos. En esa misma época, el astrónomo y matemático holandés Christiaan Huygens (1629-1695) descubrió que las fracciones continuas son una excelente herramienta para determinar el número de dientes que deben tener las ruedas de los engranajes de un reloj y las utilizó para la construcción de un autómata planetario. Por otro lado, el suizo Leonhard Euler (1707-1783) demostró que si un número tiene una fracción continua periódica, entonces es solución de una ecuación de segundo grado con coeficientes enteros. El recíproco de este resultado se debe a Joseph-Louis de Lagrange (1736- 
1813), véase teorema 1.3. Entre otras muchas aplicaciones, el también astrónomo y matemático alemán Johann Heinrich Lambert (1728-1777) las utilizó para demostrar la irracionalidad de $\pi$.

Algunos resultados de la teoría de los números son fáciles de enunciar y probar; otros son fáciles de enunciar, pero difíciles de probar, y algunas proposiciones son difíciles de comprender y difíciles de comprobar; todo esto hace que la teoría de números ocupe una posición peculiar con respecto de las distintas ramas de la matemática, por su reputación de ser difícil y estar revestida de un aura de cierto misterio, que a la vez la hace ser más interesante.

La teoría de números es fundamental para el entrenamiento inicial de todo matemático; desde el comienzo su esquema es coherente, riguroso y de extrema profundidad, las ideas fluyen y la imaginación se potencia a partir de pocas definiciones y teoremas.

\subsection{Fracciones continuas}

\section{Definición 1.1}

Una fracción continua generalizada es una expresión de la forma:

$$
\begin{array}{r}
a_{1}+\frac{b_{1}}{a_{2}+\frac{b_{2}}{a_{3}+\cdots}} \\
\cdots+\frac{b_{n-2}}{a_{n-1}+\frac{b_{n-1}}{a_{n}}}
\end{array}
$$

donde los $a_{i}$ y $b_{i}$, para $i=1,2, \ldots, n$, son números reales o números complejos.

\section{Ejemplo 1.1}

Son fracciones continuas generalizadas las expresiones $3+\frac{\pi}{-4+\frac{\sqrt{5}}{4}} y \frac{1}{6+\frac{6}{4+\frac{1}{3}}}$.

\section{Definición 1.2}

En el caso particular de las fracciones continuas en donde cada $b_{i}=1$, todos los $a_{i}$ son números enteros y para $i \geq 2$ los $a_{i}$ son positivos ( $a_{1}$ puede ser negativo o cero), la fracción se llamará 
fracción continua simple y en este caso se denotará por

$$
\begin{array}{r}
{\left[a_{1} ; a_{2}, a_{3}, \ldots, a_{n}\right]=a_{1}+\frac{1}{a_{2}+\frac{1}{a_{3}+\cdots}}} \\
\cdots+\frac{1}{a_{n-1}+\frac{1}{a_{n}}}
\end{array}
$$

Los valores $a_{i}$ se conocen como los términos de la fracción continua. En particular se tiene que $\left[a_{1}\right]=a_{1}$. Además, se dice que la fracción continua es finita si la cantidad de términos es finita; en caso contrario, se dice que la fracción continua es infinita y de la misma forma, una fracción continua simple infinita se denotará por

$$
\begin{gathered}
{\left[a_{1} ; a_{2}, a_{3}, \ldots, a_{n}, \ldots\right]=a_{1}+\frac{1}{a_{2}+\frac{1}{a_{3}+\cdots}}} \\
\cdots+\frac{1}{a_{n-1}+\frac{1}{a_{n}+\cdots}}
\end{gathered}
$$

Por último, para nuestro propósito en este artículo se asume que toda fracción continua simple infinita representa un número real.

Observe como, en los siguientes ejemplos, se encuentran las fracciones continuas simples asociadas con números racionales y el proceso inverso, es decir, dada la fracción continua se encuentra el racional que esta representa, tanto para racionales positivos como para negativos.

\section{Ejemplo 1.2}

Para encontrar la fracción racional asociada a la fracción continua simple $[2 ; 5,2,3]$, basta calcular:

$$
2+\frac{1}{5+\frac{1}{2+\frac{1}{3}}}=2+\frac{1}{5+\frac{1}{\frac{7}{3}}}=2+\frac{1}{5+\frac{3}{7}}=2+\frac{1}{\frac{38}{7}}=\frac{83}{38}
$$

por lo que $[2 ; 5,2,3]=\frac{83}{38}$. 


\section{Ejemplo 1.3}

Para encontrar la fracción continua simple asociada a $\frac{88}{25}$, basta ver que:

$$
\frac{88}{25}=3+\frac{13}{25}=3+\frac{1}{\frac{25}{13}}=3+\frac{1}{1+\frac{12}{13}}=3+\frac{1}{1+\frac{1}{\frac{13}{12}}}=3+\frac{1}{\mathbf{1}+\frac{1}{\mathbf{1}+\frac{1}{\mathbf{1 2}}}}
$$

por lo que $\frac{88}{25}=[3 ; 1,1,12]$.

\section{Ejemplo 1.4}

Para expresar $\frac{19}{11}$ como una fracción continua simple, se procede de la misma forma y se obtiene que

$$
\frac{19}{11}=\mathbf{1}+\frac{1}{\mathbf{1}+\frac{1}{2+\frac{1}{1+\frac{1}{2}}}}
$$

por lo que $\frac{19}{11}=[1 ; 1,2,1,2]$.

\section{Ejemplo 1.5}

Para encontrar la fracción continua simple asociada al racional negativo $-\frac{26}{47}$ :

$$
-\frac{26}{47}=-1+\frac{21}{47}=-1+\frac{1}{2+\frac{1}{4+\frac{1}{5}}}
$$

por lo que $-\frac{26}{47}=[-1 ; 2,4,5]$.

\section{Teorema 1.1}

Si $x$ es un número racional, $x$ se puede representar como una única fracción continua simple finita.

Demostración. Sea $x=\frac{p}{q}$ con $q>0$, por el algoritmo de la división existen $a_{1}, r_{1}$ tales que $\frac{p}{q}=a_{1}+\frac{r_{1}}{q}$ con $0<r_{1}<q$, además, $a_{1}+\frac{r_{1}}{q}=a_{1}+\frac{1}{\frac{q}{r_{1}}}$, de nuevo existen $a_{2}, r_{2}$ tales que $\frac{q}{r_{1}}=a_{2}+\frac{r_{2}}{r_{1}}$ con $0<r_{2}<r_{1}$; al seguir este proceso, se obtiene una sucesión de residuos $r_{i}$ tales que $r_{i+1}<r_{i}$, y como son positivos, por 
el principio de buen ordenamiento se concluye que este proceso es finito. Así se determina la fracción continua $\frac{p}{q}=\left[a_{1} ; a_{2}, \ldots, a_{n}\right]$, cuando $r_{n-1}=1$.

\section{Corolario 1.1}

Toda fracción continua simple infinita ${ }^{1}$ representa a un número irracional.

Demostración. Dada una fracción continua simple infinita cualquiera, esta debe representar a algún número real $x$. Por el teorema anterior, $x$ no puede ser racional, por lo que $x$ debe ser un número irracional.

\section{Definición 1.3}

Se dice que un número es un irracional cuadrático si es una solución irracional de la ecuación cuadrática $a x^{2}+b x+c=0$ con $a, b, c$ enteros y $a \neq 0$.

\section{Definición 1.4}

Una fracción continua periódica es una fracción continua simple de la forma:

$$
\left[a_{1} ; a_{2}, \ldots, a_{n}, \overline{b_{1}, b_{2}, \ldots, b_{n}}\right]
$$

donde $n$ y $m$ son enteros con $n \geq 0$ y $m \geq 1$. El periodo es la sucesión de los términos $b_{1}, b_{2}$, $\ldots, b_{n}$ que se repiten y la longitud del periodo es $m$. Si $n=0$ se dice que la fracción continua $\left[\overline{b_{1}}, b_{2}, \ldots, b_{n}\right]$ es periódica pura.

\section{Teorema 1.2}

Toda fracción continua periódica representa un número irracional cuadrático.

Demostración. Véase [8], página 197. 


\section{Ejemplo 1.6}

Para encontrar el irracional representado por la fracción continua simple infinita $[1 ; \overline{3,2}]$, se puede proceder como sigue. Sea $x=[1 ; \overline{3,2}]$, es decir

$$
x=1+\frac{1}{3+\frac{1}{2+\frac{1}{3+\frac{1}{2+\cdots}}}}
$$

por lo que $x$ satisface

$$
x-1=\frac{1}{3+\frac{1}{2+\left(\frac{1}{3+\frac{1}{2+\cdots}}\right)}}=\frac{1}{3+\frac{1}{2+(x-1)}}
$$

así, al resolver la ecuación $x-1=\frac{1}{3+\frac{1}{2+(x-1)}}$, se obtiene que sus soluciones son $\pm \sqrt{\frac{5}{3}}$;

como $x$ es positivo, se concluye que $[1 ; \overline{3,2}]=\sqrt{\frac{5}{3}}$.

Teorema 1.3 (De Lagrange).

Todo número irracional cuadrático se puede representar como una fracción continua simple infinita periódica.

La demostración del teorema anterior se omite por su complejidad. Note que este teorema de Lagrange es el recíproco del teorema 1.2, y ambos resultados nos permiten concluir que los irracionales cuadráticos son los únicos números reales que poseen representación en forma de fracción continua periódica.

\section{Ejemplo 1.7}

Para expresar $\sqrt{11}$ como fracción continua simple infinita, se oserva que $3<\sqrt{11}<4$, por lo que se puede escribir

$$
\sqrt{11}=3+(\sqrt{11}-3)=3+\frac{1}{\frac{1}{\sqrt{11}-3}}
$$


al racionalizar $\frac{1}{\sqrt{11}-3}$, se obtiene $\frac{\sqrt{11}+3}{2}$, observe que $3<\frac{\sqrt{11}+3}{2}<4$,

$$
\begin{aligned}
3+\frac{1}{\frac{1}{\sqrt{11}-3}}=3+\frac{1}{\frac{\sqrt{11}+3}{2}} & =3+\frac{1}{3+\frac{\sqrt{11}-3}{2}} \\
& =3+\frac{1}{3+\frac{1}{\frac{2}{\sqrt{11}-3}}} \\
& =3+\frac{1}{3+\frac{1}{\sqrt{11}+3}} \\
& =3+\frac{1}{3+\frac{1}{6+(\sqrt{11}-3)}}
\end{aligned}
$$

note que el término $\sqrt{11}-3$ se repite, por lo que de nuevo el ciclo inicia como en (1.1), y se concluye que $\sqrt{11}=[3 ; \overline{3,6}$. Es importante notar de que la convergencia de dicha fracción continua la garantiza el teorema 1.2.

\section{Ejemplo 1.8}

Si $p$ es un entero positivo, se cumple que la fracción continua asociada a la raíz $\sqrt{p^{2}+1}$ es $[p ; \overline{2 p}]$. Para verificarlo, basta desarrollar, como en el ejemplo 1.2, para obtener

$$
\begin{aligned}
\sqrt{p^{2}+1}=p+\left(\sqrt{p^{2}+1}-p\right) & =p+\left(\sqrt{p^{2}+1}-p\right) \cdot \frac{\sqrt{p^{2}+1}+p}{\sqrt{p^{2}+1}+p} \\
& =p+\frac{1}{\sqrt{p^{2}+1}+p} \\
& =p+\frac{1}{2 p+\left(\sqrt{p^{2}+1}-p\right)} \\
& =p+\frac{1}{2 p+\frac{1}{2 p+\left(\sqrt{p^{2}+1}-p\right)}}
\end{aligned}
$$

y a partir de ella, se observa que se inicia el periodo y se obtiene que $\sqrt{p^{2}+1}=[p ; \overline{2 p}]$.

Se puede comprobar, como en el ejemplo anterior, que si $p$ es un entero positivo, la fracción continua asociada a la raíz $\sqrt{p^{2}+2}$ es $[p ; \overline{p, 2 p}]$. 


\section{Ejemplo 1.9}

En la siguiente tabla se muestran las fracciones continuas para $\sqrt{n}$ para $n \leq 51$.

\begin{tabular}{|c|c|c|c|}
\hline$n$ & $\sqrt{n}$ & $n$ & $\sqrt{n}$ \\
\hline 2 & {$[1 ; \overline{2}]$} & 27 & {$[5 ; \overline{5,10}$} \\
\hline 3 & {$\left[1 ; \frac{\lrcorner}{1,2}\right]$} & 28 & {$[5 ; 3,2,3,10]$} \\
\hline 4 & {$[2]$} & 29 & {$[5 ; 2,1,1,2,10]$} \\
\hline 5 & {$[2 ; \overline{4}]$} & 30 & {$[5 ; 2,10]$} \\
\hline 6 & {$[2 ; 2,4]$} & 31 & {$[5 ; 1,1,3,5,3,1,1,10]$} \\
\hline 7 & {$[2 ; 1,1,1,4]$} & 32 & {$[5 ; \overline{1,1,1,10}]$} \\
\hline 8 & {$[2 ; \overline{1,4}]$} & 33 & {$[5 ; 1,2,1,10]$} \\
\hline 9 & {$[3]$} & 34 & {$[5 ; 1,4,1,10]$} \\
\hline 10 & {$[3 ; \overline{6}]$} & 35 & {$[5 ; 1,10$} \\
\hline 11 & {$\left[3 ; \frac{1}{3,6}\right]$} & 36 & [6] \\
\hline 12 & {$[3 ; 2,6]$} & 37 & $6 ; \overline{12}$ \\
\hline 13 & {$[3 ; 1,1,1,1,6]$} & 38 & {$[6 ; 6,12]$} \\
\hline 14 & {$[3 ; 1,2,1,6]$} & 39 & {$[6 ; \overline{4,12}]$} \\
\hline 15 & {$[3 ; \overline{1,6}]$} & 40 & {$[6 ; \overline{3,12}]$} \\
\hline 16 & [4] & 41 & {$[6 ; 2,2,12$} \\
\hline 17 & {$[4 ; \overline{8}]$} & 42 & {$[6 ; 2,12]$} \\
\hline 18 & {$[4 ; 4,8]$} & 43 & {$[6 ; 1,1,3,1,5,1,3,1,1,12]$} \\
\hline 19 & {$[4 ; 2,1,3,1,2,8]$} & 44 & {$[6 ; 1,1,1,2,1,1,1,12]$} \\
\hline 20 & {$[4 ; 2,8]$} & 45 & {$[6 ; 1,2,2,2,12]$} \\
\hline 21 & {$[4 ; \overline{1,1,2,1,1,8}]$} & 46 & {$[6 ; 1,3,1,1,2,6,2,1,1,3,1,12]$} \\
\hline 22 & {$[4: 1,2,4,2,1,8]$} & 47 & {$[6: 15,1,12]$} \\
\hline 23 & {$[4 ; 1,3,1,8]$} & 48 & {$[6 ; 1,12]$} \\
\hline 24 & {$[4 ; \overline{1,8}]$} & 49 & {$[7]$} \\
\hline 25 & {$[5]$} & 50 & {$[7: \overline{14}]$} \\
\hline 26 & {$[5 ; \overline{10}$} & 51 & {$[7 ; 7,14$} \\
\hline
\end{tabular}

\section{Ejemplo 1.10}

Para expresar $\sqrt{39}-2$ como fracción continua simple infinita, se puede proceder como en el ejemplo 1.2; sin embargo, haciendo uso de la tabla anterior, y dado que $\sqrt{39}=[6 ; \overline{4,12}]$, se obtiene que $\sqrt{39}-2=[4 ; \overline{4,12}]$.

$\mathrm{Al}$ observar con detenimiento la forma de la fracción continua periódica asociada a los valores de las raíces $\sqrt{k}$, con $k=8,15,24,35$ y 48 en la tabla anterior, es posible conjeturar que $\sqrt{p^{2}-1}=[p ; \overline{1,2 p}]$, resultado que es fácil de verificar. De la misma forma, al observar los valores de las raíces $\sqrt{k}$, con $k=6,12,20,30$ y 42 , es posible conjeturar y probar que $\sqrt{p^{2}+p}=[p ; \overline{2,2 p}]$. 


\section{Definición 1.5}

Se definen los convergentes $c_{k}$ de la fracción continua simple infinita $\left[a_{1} ; a_{2}, a_{3}, \ldots\right]$, para todo $k$ entero positivo, como las fracciones finitas

$$
\begin{aligned}
c_{1}= & {\left[a_{1}\right] } \\
c_{2}= & {\left[a_{1} ; a_{2}\right] } \\
\vdots & \vdots \\
c_{k}= & {\left[a_{1} ; a_{2}, a_{3}, \ldots, a_{k}\right] }
\end{aligned}
$$

En el caso de que la fracción continua simple sea finita $\left[a_{1} ; a_{2}, a_{3}, \ldots, a_{n}\right]$ sus $n$ convergentes serán las fracciones finitas: $c_{1}=\left[a_{1}\right], c_{2}=\left[a_{1} ; a_{2}\right], \ldots, c_{n}=\left[a_{1} ; a_{2}, a_{3}, \ldots, a_{n}\right]$.

\section{Ejemplo 1.11}

Para calcular los convergentes de la fracción continua $[2 ; 3,2,5]$, de acuerdo con la definición, se tiene que $c_{1}=[2]=2, c_{2}=[2 ; 3]=2+\frac{1}{3}=\frac{7}{3}, c_{3}=[2 ; 3,2]=2+\frac{1}{3+\frac{1}{2}}=\frac{16}{7}$ y por último, $c_{4}=$ $[2 ; 3,2,5]=2+\frac{1}{3+\frac{1}{2+\frac{1}{5}}}=\frac{87}{38}$.

\section{Ejemplo 1.12}

Para calcular los primeros cuatro convergentes de la fracción continua infinita $[4 ; \overline{3,2}]$, se observa que en este caso, se tiene $c_{1}=[4]=4, c_{2}=[4 ; 3]=4+\frac{1}{3}=\frac{13}{3}, c_{3}=[4 ; 3,2]=4+\frac{1}{3+\frac{1}{2}}=\frac{30}{7}$ y por último, $c_{4}=[4 ; 3,2,3]=4+\frac{1}{3+\frac{1}{2+\frac{1}{3}}}=\frac{103}{24}$.

Es claro que para la fracción continua simple $\left[a_{1} ; a_{2}, a_{3}, \ldots\right]$, finita o infinita, cada convergente $c_{k}$ de ella es un racional; por consiguiente, es posible afirmar que $c_{k}=\frac{p_{k}}{q_{k}}, \forall k \in \mathbb{N}$, con $p_{k}$ y $q_{k}$ enteros tales que $\operatorname{mcd}\left(p_{k}, q_{k}\right)=1$. De hecho, se puede encontrar una fórmula para dichos enteros, iniciando con $n=1$ :

$$
c_{1}=\left[a_{1}\right]=\frac{a_{1}}{1}
$$

por lo que $p_{1}=a_{1}$ y $q_{1}=1$, ahora para $n=2$ :

$$
c_{2}=\left[a_{1} ; a_{2}\right]=a_{1}+\frac{1}{a_{2}}=\frac{a_{1} a_{2}+1}{a_{2}}
$$


por lo que $p_{2}=a_{1} a_{2}+1$ y $q_{2}=a_{2}$, ahora para $n=3$ :

$$
\begin{aligned}
c_{3}=\left[a_{1} ; a_{2}, a_{3}\right]=a_{1}+\frac{1}{a_{2}+\frac{1}{a_{3}}} & =\frac{a_{3} a_{2} a_{1}+a_{1}+a_{3}}{a_{3} a_{2}+1} \\
& =\frac{a_{3}\left(a_{2} a_{1}+1\right)+a_{1}}{a_{3} a_{2}+1} \\
& =\frac{a_{3} p_{2}+p_{1}}{a_{3} q_{2}+q_{1}}
\end{aligned}
$$

con lo cual $p_{3}=a_{3} p_{2}+p_{1}$ y $q_{3}=a_{3} q_{2}+q_{1}$.

Siguiendo el razonamiento anterior se obtiene el siguiente teorema.

Teorema 1.4

Si $c_{n}=\frac{p_{n}}{q_{n}}$ es el $n$-ésimo convergente de la fracción continua simple $\left[a_{1} ; a_{2}, a_{3}, \ldots\right]$, entonces $p_{1}=a_{1}$ y $q_{1}=1$ para $n=1 ; p_{2}=a_{2} p_{1}+1$ y $q_{2}=a_{2}$ para $n=2 ; \mathrm{y} \forall n \geq 3$ se cumple que:

$$
\begin{aligned}
& p_{n}=a_{n} p_{n-1}+p_{n-2} \\
& q_{n}=a_{n} q_{n-1}+q_{n-2}
\end{aligned}
$$

Demostración. Del resultado anterior, se cumple para $n=1$ y $n=2$. Para $n \geq 3$ se utiliza el método de inducción:

- Para $n=3$ es válido, pues es el desarrollo de $c_{3}$ dado en (1.3).

- Se asume que la proposición es válida para n, es decir, se asume como hipótesis de inducción (H.I.) las identidades (1.4) y (1.5).

- Se debe probar que la proposición es válida para $n+1$; para ello, observe que:

$$
c_{n+1}=\left[a_{1} ; a_{2}, a_{3}, \ldots, a_{n}, a_{n+1}\right]=\left[a_{1} ; a_{2}, a_{3}, \ldots, a_{n-1}, a_{n}+\frac{1}{a_{n+1}}\right]
$$

pues, aunque $a_{n}+\frac{1}{a_{n+1}}$ no es necesariamente entero, la notación lo permite y se tiene que:

$$
\begin{aligned}
c_{n+1} & \stackrel{\text { H.I. }}{=} \frac{\left(a_{n}+\frac{1}{a_{n+1}}\right) p_{n-1}+p_{n-2}}{\left(a_{n}+\frac{1}{a_{n+1}}\right) q_{n-1}+q_{n-2}} \\
& =\frac{a_{n+1}\left(a_{n} p_{n-1}+p_{n-2}\right)+p_{n-1}}{a_{n+1}\left(a_{n} q_{n-1}+q_{n-2}\right)+q_{n-1}} \stackrel{\text { H.I. }}{=} \frac{a_{n+1} p_{n}+p_{n-1}}{a_{n+1} q_{n}+q_{n-1}}
\end{aligned}
$$

por lo tanto $p_{n+1}=a_{n+1} p_{n}+p_{n-1}$ y $q_{n+1}=a_{n+1} q_{n}+q_{n-1}$.

Con lo cual, las fórmulas (1.4) y (1.5) son válidas para todo $n \geq 3$. 


\section{Ejemplo 1.13}

Para calcular los convergentes de $[3 ; 2,2,4,3,2,4]$ se utilizan las fórmulas recursivas (1.4) y (1.5); para ello es conveniente construir la siguiente tabla:

\begin{tabular}{|l||c|c|c|c|c|c|c|}
\hline$i$ & 1 & 2 & 3 & 4 & 5 & 6 & 7 \\
\hline$a_{i}$ & 3 & 2 & 2 & 4 & 3 & 2 & 4 \\
\hline$p_{i}$ & 3 & 7 & 17 & 75 & 242 & 559 & 2478 \\
\hline$q_{i}$ & 1 & 2 & 5 & 22 & 71 & 164 & 727 \\
\hline$c_{i}$ & 3 & $\frac{7}{2}$ & $\frac{17}{5}$ & $\frac{75}{22}$ & $\frac{242}{71}$ & $\frac{559}{164}$ & $\frac{2478}{727}$ \\
\hline
\end{tabular}

\section{Ejemplo 1.14}

Para calcular los convergentes $c_{4}$ y $c_{6}$ de $[5 ; \overline{3,4}]$ se procede como en el ejemplo anterior, se construye la tabla:

\begin{tabular}{|l||c|c|c|c|c|c|}
\hline$i$ & 1 & 2 & 3 & 4 & 5 & 6 \\
\hline$a_{i}$ & 5 & 3 & 4 & 3 & 4 & 3 \\
\hline$p_{i}$ & 5 & 16 & 69 & 223 & 961 & 3106 \\
\hline$q_{i}$ & 1 & 3 & 13 & 42 & 181 & 585 \\
\hline$c_{i}$ & & & & $\frac{223}{42}$ & & $\frac{3106}{585}$ \\
\hline
\end{tabular}

Finalmente, $c_{4}=\frac{223}{42}$ y $c_{6}=\frac{3106}{585}$.

\section{Teorema 1.5}

Si el $n$-ésimo convergente asociado a la fracción continua simple $\left[a_{1} ; a_{2}, a_{3}, \ldots\right]$ es $c_{n}=\frac{p_{n}}{q_{n}}$, entonces $\forall n \geq 2$ se cumple que

$$
p_{n} q_{n-1}-p_{n-1} q_{n}=(-1)^{n}
$$

Demostración. Se utiliza el método de inducción:

- Para $n=2$ es válido, pues de (1.2) se tiene:

$$
p_{2} q_{1}-p_{1} q_{2}=\left(a_{2} a_{1}+1\right) \cdot 1-a_{1} \cdot a_{2}=1=(-1)^{2}
$$

- Se supone que la proposición es válida para $n$, es decir, se toma como hipótesis de inducción (H.I.) la expresión (1.6).

- Ahora se probará la validez de la proposición para $n+1$; así:

$$
\begin{aligned}
p_{n+1} q_{n}-p_{n} q_{n+1} & =\left(a_{n+1} p_{n}+p_{n-1}\right) q_{n}-p_{n}\left(a_{n+1} q_{n}+q_{n-1}\right) \\
& =a_{n+1} p_{n} q_{n}+p_{n-1} q_{n}-p_{n} a_{n+1} q_{n}-p_{n} q_{n-1} \\
= & -\left(p_{n} q_{n-1}-p_{n-1} q_{n}\right) \\
\stackrel{\text { H.I. }}{=} & -(-1)^{n}=(-1)^{n+1}
\end{aligned}
$$


con lo cual, el resultado (1.6) es válido para todo $n \geq 2$.

\section{Corolario 1.2}

Si el $n$-ésimo convergente asociado a la fracción continua simple $\left[a_{1} ; a_{2}, a_{3}, \ldots\right]$ es $c_{n}=\frac{p_{n}}{q_{n}}$, entonces $\forall n \geq 2$ se cumple que

$$
c_{n}-c_{n-1}=\frac{(-1)^{n}}{q_{n} q_{n-1}}
$$

Demostración. Utilizando el teorema anterior, basta ver:

$$
c_{n}-c_{n-1}=\frac{p_{n}}{q_{n}}-\frac{p_{n-1}}{q_{n-1}}=\frac{p_{n} q_{n-1}-p_{n-1} q_{n}}{q_{n} q_{n-1}}=\frac{(-1)^{n}}{q_{n} q_{n-1}}
$$

En ocasiones es útil recurrir al concepto de determinante para recordar el resultado, dado en el teorema 1.5 sobre los convergentes sucesivos. De esta forma, la igualdad (1.6) se reescribe como:

$$
\left|\begin{array}{ll}
p_{n} & p_{n-1} \\
q_{n} & q_{n-1}
\end{array}\right|=(-1)^{n}
$$

Este resultado se utilizará en la sección 1.3 para resolver ecuaciones diofánticas y en la sección 1.4 para encontrar algunos criterios de divisibilidad.

\section{Teorema 1.6}

Si el $n$-ésimo convergente asociado a la fracción continua simple $\left[a_{1} ; a_{2}, a_{3}, \ldots\right]$ es $c_{n}=\frac{p_{n}}{q_{n}}$, entonces $\forall n \geq 3$ se cumple que

$$
c_{n}-c_{n-2}=\frac{(-1)^{n-1} a_{n}}{q_{n} q_{n-2}}
$$

Demostración. Primero se calcula:

$$
\begin{aligned}
p_{n} q_{n-2}-p_{n-2} q_{n} & =\left(a_{n} p_{n-1}+p_{n-2}\right) q_{n-2}-p_{n-2}\left(a_{n} q_{n-1}+q_{n-2}\right) \\
& =a_{n} p_{n-1} q_{n-2}+p_{n-2} q_{n-2}-a_{n} p_{n-2} q_{n-1}-p_{n-2} q_{n-2} \\
& =a_{n}\left(p_{n-1} q_{n-2}-p_{n-2} q_{n-1}\right) \\
& =(-1)^{n-1} a_{n}
\end{aligned}
$$

al dividir por $q_{n} q_{n-2}$, se obtiene:

$$
\begin{aligned}
& \frac{p_{n} q_{n-2}-p_{n-2} q_{n}}{q_{n} q_{n-2}}=\frac{(-1)^{n-1} a_{n}}{q_{n} q_{n-2}} \\
\Rightarrow & \frac{p_{n}}{q_{n}}-\frac{p_{n-2}}{q_{n-2}}=\frac{(-1)^{n-1} a_{n}}{q_{n} q_{n-2}} \\
\Rightarrow & c_{n}-c_{n-2}=\frac{(-1)^{n-1} a_{n}}{q_{n} q_{n-2}}
\end{aligned}
$$

como se quería probar. 


\section{Corolario 1.3}

La subsucesión de los convergentes de índice par de toda fracción continua simple infinita es decreciente, mientras que la subsucesión de los convergentes de índice impar es creciente. Además, todo convergente impar es menor que todo convergente par.

Demostración. Dado que $a_{n}, q_{n}$ y $q_{n-2}$ son positivos, de la identidad (1.10) se tiene que $c_{n}-c_{n-2}<0$ si $n$ es par y $c_{n}-c_{n-2}>0$ si $n$ es impar. Por otro lado, para $s$ y $t$ números enteros positivos cualesquiera:

- Si $s<t$, se tiene que $c_{2 s}>c_{2 t}$ pues es decreciente para los pares. Además, se sabe que $c_{2 t}>c_{2 t-1}$, con lo cual $c_{2 s}>c_{2 t-1}$.

- Si $s>t$, se tiene que $c_{2 s-1}>c_{2 t-1}$ pues es creciente para los impares. Además, se sabe que $c_{2 s}>c_{2 s-1}$, con lo cual $c_{2 s}>c_{2 t-1}$.

- Si $s=t$, es claro que $c_{2 s}>c_{2 t-1}$.

con lo que $c_{2 s}>c_{2 t-1}$ en cualquier caso y con ello todo convergente impar es menor que todo convergente par.

El siguiente ejemplo se relaciona con los llamados números metálicos, los cuales son una familia de números en donde el más conocido de sus miembros es el número de oro; sin embargo, en ella existen los de plata, bronce, níquel, cobre, entre otros. Las aplicaciones de ellos son variadas, por ejemplo, a lo largo de la historia han sido utilizados por varias culturas como base de proporciones; sin embargo, en la actualidad se han utilizado en algunos campos como los sistemas no lineales y la teoría del caos.

\section{Ejemplo 1.15}

En general, al considerar los valores enteros positivos de $b$, las soluciones positivas de la ecuación cuadrática $x^{2}-b x-1=0$ son elementos del conjunto de los números metálicos. Para $b=1$, por ejemplo, se obtiene el número de oro $x=\frac{1+\sqrt{5}}{2}$; para $b=2$ se obtiene el llamado número de plata $x=1+\sqrt{2}$; para $b=3$ se obtiene el número de bronce $x=\frac{3+\sqrt{13}}{2}$. Lo interesante de esta ecuación es que al despejar se obtiene $x=b+\frac{1}{x}$, y al sustituir reiteradamente este valor de $x$ en el término de la derecha se obtiene:

$$
\begin{aligned}
x & =b+\frac{1}{b+\frac{1}{x}} \\
& =b+\frac{1}{b+\frac{1}{b+\frac{1}{x}}}
\end{aligned}
$$

y con ella una expresión para los números metálicos en fracción continua periódica de la forma $x=[b ; \bar{b}]$. 


\section{Teorema 1.7}

Si $a, b$ son enteros positivos tales que $b^{2}+4 a$ no es cuadrado perfecto y $a \mid b$ entonces, $\left[\frac{b}{a} ; \bar{b}, \frac{b}{a}\right]$ es raíz de la ecuación $a x^{2}-b x-1=0$.

Demostración. Sea $x=\left[\frac{b}{a} ; \bar{b}, \frac{b}{a}\right]$, es decir, $x=\frac{b}{a}+\frac{1}{b+\frac{1}{\frac{b}{a}+\frac{1}{b+\cdots}}}$ y se obtiene que

$$
\begin{aligned}
x & =\frac{b}{a}+\frac{1}{b+\frac{1}{x}} \\
& =\frac{b}{a}+\frac{x}{b x+1}=\frac{b^{2} x+b+a x}{a b x+a}
\end{aligned}
$$

es decir, la identidad $x=\frac{b^{2} x+b+a x}{a b x+a}$ y al despejar se obtiene $a x^{2}-b x-1=0$ cuyas soluciones son irracionales si su discriminante $b^{2}+4 a$ no es un cuadrado perfecto que es lo que se tiene pues al ser periódico, debe ser irracional.

\section{Ejemplo 1.16}

El número $e$ tiene su representación en fracción continua simple no periódica:

$$
e=[2 ; 1,2,1,1,4,1,1,6,1,1,8,1,1,10,1, \ldots]
$$

su representación mantiene un patrón en donde se observa el cuasi-periodo en grupos de tres de la forma $1,2 p, 1$ para $p \geq 1$.

\section{Ejemplo 1.17}

El número $\pi$ tiene su representación en fracción continua simple no periódica:

$$
\pi=[3 ; 7,15,1,292,1,1,2, \ldots]
$$

los convergentes son $c_{1}=[3]=3, c_{1}=[3 ; 7]=\frac{22}{7}, c_{3}=[3 ; 7,15]=\frac{333}{106}, c_{4}=[3 ; 7,15,1]=\frac{355}{113}, \ldots$ Es interesante que el segundo convergente $c_{2}=[3 ; 7]=\frac{22}{7}$ sea la aproximación que utilizó Arquímedes en sus trabajos. 


\section{Ejemplo 1.18}

Una representación de $\pi$ como fracción continua generalizada es:

$$
\pi=\frac{4}{1+\frac{1}{3+\frac{4}{5+\frac{9}{7+\frac{16}{9+\frac{25}{\ddots}}}}}}
$$

otra representación es:

$$
\pi=3+\frac{1}{6+\frac{9}{6+\frac{25}{6+\frac{49}{6+\frac{81}{6+\frac{121}{\ddots}}}}}}
$$

Ambas son interesantes, pues a pesar de ser trascendente, su representación mantiene un patrón.

\section{Ejemplo 1.19}

La siguiente identidad es original del gran matemático de la India, Srinivasa Ramanujan (18871920):

$$
1+\frac{1}{1 \cdot 3}+\frac{1}{1 \cdot 3 \cdot 5}+\frac{1}{1 \cdot 3 \cdot 5 \cdot 7}+\cdots+\frac{1}{1+\frac{1}{1+\frac{2}{1+\frac{3}{1+\frac{4}{\ddots}}}}}=\sqrt{\frac{\pi e}{2}}
$$

con gran belleza, esta fórmula involucra la suma de una fraccción continua generalizada infinita y una serie que se complementan armónicamente, cuyo resultado contiene dos de los números trascendentes más representativos.

\subsection{Ecuaciones diofánticas}

En el libro Elementos de álgebra, escrito por Euler en 1770, se enucia el siguiente problema: 
"Dos personas tienen 100 huevos entre ambas. Una le dijo a la otra -Cuando cuento los huevos que tengo por grupos de ocho, me sobran 7-. La segunda comentó: -Cuando yo cuento los que tengo por grupos de diez, también me sobran 7-. ¿Cuántos huevos tenía cada persona?"

Como el número de huevos que tiene la primera persona dividido por 8 da residuo 7 , esta cantidad es de la forma $8 x+7$ donde $x$ es el cociente de dicha división; de la misma forma, la cantidad de la segunda persona es de la forma $10 y+7$. Además, se sabe que su suma es 100 , por lo que $8 x+7+$ $10 y+7=100$, es decir, luego de simplificar se debe resolver la ecuación $4 x+5 y=43$; al despejar $x$ :

$$
x=\frac{43-5 y}{4}
$$

de esta forma, es claro que tanto $x$ como $y$ deben ser positivos, y además $x$ debe ser entero, por lo que se deben escoger valores de $y$ para los cuales $43-5 y$ sea divisible por 4 . Con un poco de inspección es posible encontrar que, por ejemplo, si $y=7$, entonces $x=2$, y una posible solución al problema sería que la primera persona tiene 23 huevos y la segunda 77 . Luego se verá que hay otra solución a este problema. En general se presentan varios métodos para resolver ecuaciones de este tipo, es decir, ecuaciones lineales donde interesan sus soluciones enteras.

\section{Definición 1.6}

Toda ecuación lineal de la forma

$$
a_{1} x_{1}+a_{2} x_{2}+\cdots+a_{n} x_{n}=c
$$

donde los $a_{i}$ y $c$ son enteros y los posibles valores de $x_{i}$ soluciones de la ecuación, son números enteros, se llamará ecuación diofántica.

\section{Teorema 1.8}

La ecuación diofántica lineal $a x+b y=c$ tiene solución si y solo si $\operatorname{mcd}(a, b) \mid c$.

Demostración. Sea $g=\operatorname{mcd}(a, b)$, por la definición se sabe que existen $a^{\prime}, b^{\prime}$ enteros tales que $a=g a^{\prime}$ y $b=g b^{\prime}$; así:

$" \Rightarrow$ Suponga que existen $x, y \in \mathbb{Z}$ que satisfacen $a x+b y=c$; entonces:

$$
a x+b y=g a^{\prime} x+g b^{\prime} y=g\left(a^{\prime} x+b^{\prime} y\right)=c
$$

con lo cual se tiene que $g \mid c$, pues $g$ es factor de $c$.

$" \Leftarrow "$ Suponga que $g \mid c$, se tiene que $c=g c^{\prime}$ para algún $c^{\prime}$ entero. Por la Identidad de Bézout ${ }^{2}$, existen $x^{\prime}, y^{\prime}$ tales que $a x^{\prime}+b y^{\prime}=g$, por lo que al multiplicar por $c^{\prime}$ se obtiene

$$
a\left(x^{\prime} c^{\prime}\right)+b\left(y^{\prime} c^{\prime}\right)=g c^{\prime}=c
$$

${ }^{2}$ Esta garantiza que para $a$ y $b$ enteros no nulos, existen $s$ y $t$ enteros tales que $\operatorname{mcd}(a, b)=a s+b t$. 
y se ha probado que $\left(x^{\prime} c^{\prime}, y^{\prime} c^{\prime}\right)$ es solución entera de la ecuación.

con lo cual se ha probado la equivalencia deseada.

El teorema anterior garantiza la existencia de solución si $\operatorname{mcd}(a, b) \mid c$; por lo tanto, al resolver una ecuación de este tipo, se puede dividir la ecuación por $\operatorname{mcd}(a, b)$, de manera que los coeficientes sean coprimos.

\section{Teorema 1.9}

Si $\operatorname{mcd}(a, b)=1$ y además $\left(x_{0}, y_{0}\right)$ es una solución de la ecuación diofántica lineal

$$
a x+b y=c
$$

entonces, $(x, y)$ es solución de la ecuación (1.11) si y solo si es de la forma

$$
x=x_{0}-b n, \quad y=y_{0}+a n, \quad n \in \mathbb{Z}
$$

Demostración. Se debe probar que $(x, y)$ es solución de (1.11) si y solo si es de la forma (1.12):

$" \Rightarrow "$ Si se supone que $(x, y)$ es solución, se tiene que $a x+b y=c$ es verdadera; como además $\left(x_{0}, y_{0}\right)$ es solución, se tiene $a x_{0}+b y_{0}=c$. Restando estas igualdades,

$$
a x-a x_{0}+b y-b y_{0}=0
$$

al despejar se obtiene

$$
y-y_{0}=\frac{a\left(x_{0}-x\right)}{b}
$$

es claro que $y-y_{0}$ es entero, por lo que $b \mid a\left(x_{0}-x\right)$, pero como $\operatorname{mcd}(a, b)=1$, por el lema de Euclides se deduce que $b \mid\left(x_{0}-x\right)$; así, existe $n \in \mathbb{Z}$ tal que $x_{0}-x=b n$, es decir, $x=x_{0}-b n, \mathrm{y}$ al sustituir este valor de $x$ en (1.13) se tiene que $y=y_{0}+\frac{a b n}{b}=y_{0}+a n$.

$" \Leftarrow "$ Si se supone que $x^{\prime}, y^{\prime}$ son de la forma (1.12), existe $n \in \mathbb{Z}$ tal que $x^{\prime}=x_{0}-b n, y^{\prime}=y_{0}+a n, \mathrm{y}$ como $\left(x_{0}, y_{0}\right)$ es solución, se verifica

$$
a x^{\prime}+b y^{\prime}=a x_{0}-a b n+b y_{0}+b a n=a x_{0}+b y_{0}=c
$$

por lo tanto, $\left(x^{\prime}, y^{\prime}\right)$ es una solución.

Así, se probó que si $(x, y)$ es solución, entonces es de la forma (1.12) y viceversa.

El teorema anterior proporciona una fórmula para la solución general de una ecuación diofántica en dos variables, a partir de una solución particular denotada por $\left(x_{0}, y_{0}\right)$; así, para aplicar dicho teorema es necesario conocer o determinar una solución particular. Para ello existen varios métodos, uno que 
utiliza el algoritmo de la división, otro utilizando las fracciones continuas y un tercero con base en las congruencias numéricas. En los siguientes ejemplos se utilizará un procedimiento que involucra a los convergentes de las fracciones continuas.

Específicamente, se calcula la fracción continua asociada a $\frac{a}{b}$, donde $a$ y $b$ son los coeficientes de la ecuación diofántica dada. Se sabe que si $\frac{a}{b}=\left[a_{1} ; a_{2}, \ldots, a_{n}\right]$, para los convergentes $c_{k}=\frac{p_{k}}{q_{k}}$ se tiene que $c_{n}=\frac{a}{b}$, aplicando el resultado (1.6) dado en la página 12 se tiene:

$$
a q_{n-1}-p_{n-1} b=(-1)^{n}
$$

por lo que simplemente basta multiplicar por $(-1)^{n} c$ para obtener una solución particular de la forma:

$$
a\left[q_{n-1}(-1)^{n} c\right]+b\left[-p_{n-1}(-1)^{n} c\right]=c
$$

\section{Ejemplo 1.20}

Para resolver la ecuación diofántica $61 x+18 y=8$ utilizando el método de las fracciones continuas, primero se observa que como $\operatorname{mcd}(61,18)=1$, la ecuación tiene solución. Al aplicar las fracciones continuas se obtiene que:

$$
\frac{61}{18}=3+\frac{1}{2+\frac{1}{1+\frac{1}{1+\frac{1}{3}}}}
$$

es decir, $\frac{61}{18}=[3 ; 2,1,1,3]$ al eliminar la fracción $\frac{1}{3}$ se obtiene el convergente $[3 ; 2,1,1]$, que equivale a:

$$
3+\frac{1}{2+\frac{1}{1+\frac{1}{1}}}=\frac{17}{5}
$$

utilizando el resultado (1.6), página 12, se obtiene:

$$
61 \cdot 5-17 \cdot 18=(-1)^{5}
$$

al multiplicar la ecuación por -8 y agrupar, se obtiene la identidad:

$$
61 \cdot(-40)+18 \cdot(136)=8
$$

Así, una solución particular es $x=-40$ y $y=136$, de donde la solución general es de la forma $x=-40-18 n$ y $y=136+61 n$ con $n \in \mathbb{Z}$. 


\section{Ejemplo 1.21}

Para resolver la ecuación diofántica $418 x-131 y=3$ utilizando el mismo método, primero se encuentra que $\frac{418}{131}=[3 ; 5,4,6]$ y es claro que $c_{4}=\frac{418}{131}$ y $c_{3}=\frac{67}{21}$; del resultado (1.6) se obtiene la identidad $418 \cdot 21-131 \cdot 67=(-1)^{4}$, que al multiplicarse por 3 se transforma en

$$
418 \cdot 63-131 \cdot 201=3
$$

así, una solución particular es $x=63$ y $y=201$, de donde la solución general es de la forma $x=63+131 n$ y $y=201+418 n$ con $n \in \mathbb{Z}$.

\section{Ejemplo 1.22}

De la misma forma, se puede resolver la ecuación diofántica $10 x-14 y=62$ utilizando el método de las fracciones continuas, pero primero debemos obtener la ecuación equivalente a $5 x-7 y=$ 31; usando el método de las fracciones continuas se encuentra que $\frac{7}{5}=1+\frac{1}{2+\frac{1}{2}}$, es decir, $\frac{7}{5}=$ $c_{3}=[1 ; 2,2]$, y al eliminar el 2 se tiene $c_{2}=[1 ; 2]=\frac{3}{2}$. Al utilizar el resultado (1.6), se obtiene la nueva identidad $7 \cdot 2-5 \cdot 3=(-1)^{3}$, que al multiplicarla por -31 se transforma en:

$$
5 \cdot 93-7 \cdot 62=31
$$

así, una solución particular es $x=93$ y $y=62$, de donde la solución general es de la forma $x=93+7 n$ y $y=62+5 n$ con $n \in \mathbb{Z}$.

\subsection{Criterios de divisibilidad}

En [6] se encuentran algunos criterios de divisibilidad a partir del desarrollo de los residuos potenciales de $t$ módulo $m$, principalmente con $t=10$, por ser la base que se utiliza con mayor frecuencia. Ahora se justificarán otros criterios utilizando las propiedades de las fracciones continuas.

Si $n$ es un natural cualquiera, es claro que en base 10 se puede escribir como $n=10 a+u$ con $u$ el dígito de las unidades, donde $0 \leq u<10$ y $a$ es el número que se obtiene de suprimir a $n$ el dígito de las unidades. El criterio de la divisibilidad por 7 conocido afirma que $7 \mid n$ si y solo si $7 \mid(a-2 u)$, es decir "borre el último dígito del número $n$, luego, reste dos veces este dígito al número resultante. Si dicho número es divisible por 7 , el número original $n$ lo será también".

En general, en esta sección se tratará de encontrar un criterio de divisibilidad por $m$ de la forma $m \mid n$ si y solo si $m \mid(a+k u)$ para números escritos en base 10 . 
Para iniciar suponga que $m>10$, a partir de que $m \mid n$ y $m \mid m a$; es posible aplicar la propiedad de linealidad para que de:

$$
m \mid(k(10 a+u)+y m a)
$$

se obtenga:

$$
m|((10 k+y m) a+k u) \Longleftrightarrow m|(a+k u)
$$

es decir, se debe encontrar $k$ entero que satisfaga la ecuación diofántica:

$$
10 k+m y=1
$$

Note que puede haber varios valores enteros para $k$ y para $y$, este detalle se explica al final de esta sección en donde se observa que existen varios posibles criterios, todos equivalentes módulo $m$. Para que (1.14) tenga solución, es necesario que $\operatorname{mcd}(10, m)=1$. Si $\left[a_{1} ; a_{2}, a_{3}, \ldots, a_{n}\right]$ es la fracción continua simple finita de la fracción $\frac{m}{10}$, utilizando la identidad (1.6) se sabe que los convergentes $c_{k}=\frac{p_{k}}{q_{k}}$ satisfacen la propiedad:

$$
m q_{n-1}-10 p_{n-1}=(-1)^{n}
$$

pues en este caso, $c_{n}=\frac{m}{10}$, el último convergente es $\frac{m}{10}$.

Luego de comparar términos en las ecuaciones (1.14) y (1.15), es claro que si $n$ es par, $k=-p_{n-1}$ y si $n$ es impar, $k=p_{n-1}$. Por último, en el caso de que $m<10$, el proceso es análogo y se debe desarrollar la fracción continua de $\frac{m}{10}=0+\frac{1}{\frac{10}{m}}$.

\section{Ejemplo 1.23}

Para determinar un criterio de divisibilidad por 23, se observa que para $m=23$ se obtiene que $\frac{23}{10}=[2 ; 3,3]$, así $c_{2}=[2 ; 3]=\frac{7}{3}$, por lo que $p_{2}=7$, y dado que $n$ es impar, se obtiene que $k=7 \mathrm{y}$ el criterio para $n=10 a+u$ simbólicamente sería

$$
23|n \Longleftrightarrow 23|(a+7 u)
$$

o en palabras: "borre el último dígito del número $n$, luego, sume siete veces este dígito al número resultante. Si dicho número es divisible por 23, el número original $n$ lo será también". 


\section{Ejemplo 1.24}

Utilicemos el criterio anterior para determinar si 11338 es divisible por 23. Como el dígito de las unidades es 8 , se forma el nuevo número

$$
11338+7 \cdot 8=1133+56=1189
$$

Ahora se repite el criterio y como el dígito de las unidades es 9 , se forma el nuevo número $118+7 \cdot 9=181$. Ahora el dígito de las unidades es 1 , se forma el número $18+7=25$ que claramente no es divisible por 23; por lo que se concluye que 23 no divide a 11338 .

\section{Ejemplo 1.25}

Para determinar un criterio de divisibilidad por 7 , es claro que en este caso $m=7 \mathrm{y}$ al desarrollar la fracción continua se tiene que $\frac{7}{10}=[0 ; 1,2,3]$, así $c_{3}=[0 ; 1,2]=\frac{2}{3}$, por lo que $p_{3}=2$, y dado que $n$ es par, se obtiene que $k=-2$ y el criterio para $n=10 a+u$ simbólicamente sería

$$
7|n \Longleftrightarrow 7|(a-2 u)
$$

o en palabras: "borre el último dígito del número $n$, luego, reste dos veces este dígito al número resultante. Si este número es divisible por 7 , el número original $n$ lo será también".

\section{Ejemplo 1.26}

Utilicemos el criterio anterior para determinar si 204673 es divisible por 7. Como el dígito de las unidades es 3, se forma el nuevo número

$$
20467 \not \supset-2 \cdot 3=20467-6=20461
$$

Ahora se repite el criterio y como el dígito de las unidades es 1 , se forma el nuevo número $2046-2=2044$. Ahora el dígito de las unidades es 4 , se forma de nuevo el número $204-8=196$. Ahora el dígito de las unidades es 6 , se forma de nuevo el número $19-12=7$ que claramente es divisible por 7; por lo que se concluye que $7 \mid 204673$.

Con la notación empleada aquí, entre otros, se puede comprobar la validez de los siguientes criterios de divisivilidad:

1. $19|n \Longleftrightarrow 19|(a-17 u)$ o $19|n \Longleftrightarrow 19|(a+2 u)$, que es más fácil de aplicar.

2. $13|n \Longleftrightarrow 13|(a+4 u) \Longleftrightarrow 13 \mid(a-9 u)$

3. $47|n \Longleftrightarrow 47|(a-14 u)$ 
4. $67|n \Longleftrightarrow 67|(a-20 u)$

5. $37|n \Longleftrightarrow 37|(a-11 u)$.

\subsection{Calendarios}

En general, un calendario divide el tiempo y lo agrupa en distintos periodos o intervalos. Sobre la evolución del calendario gregoriano, que fue el resultado del seguimiento a los astros, paralelo al desarrollo matemático en donde se conjugaron tradiciones culturales heredadas o impuestas, y ajustes realizados para su perfeccionamiento, se recomienda consultar [6].

Para iniciar recuerde que la medición del paso del tiempo se ha asociado con tres ciclos astronómicos. El día como el tiempo que corresponde a una rotación de la Tierra sobre su eje; el mes como el tiempo que tarda la Luna en girar alrededor de la Tierra; visto desde la Tierra, es el tiempo entre una Luna Nueva y la siguiente, y tarda aproximadamente 29,5 días. Como tercer ciclo de referencia, el año es el tiempo que corresponde a una revolución de la Tierra alrededor del Sol.

Todos los planetas tienen su propia duración del día y del año. Mercurio, por ejemplo, es el más próximo al Sol y el más pequeño de nuestro sistema solar: su periodo de rotación (día) es de 58,7 días terrestres, y logra completar su órbita en torno al Sol (año) en tan solo 88 días terrestres, con lo cual su periodo de rotación es $\frac{2}{3}$ de su periodo de traslación; así, mientras Mercurio recorre dos veces su órbita alrededor del Sol, solo da tres vueltas sobre su propio eje, es decir, en el calendario de Mercurio hay poco menos de dos días cada año.

A Rómulo se le atribuye la creación del año de 10 meses, iniciando en martius; luego Numa Pompilio lo llevó a 12 meses. Julio César, como pontífice máximo y gobernador de Roma, a la conquista de Egipto llevó a Sosígenes, astrónomo de Alejandría, y le encomendó perfeccionar el calendario. En la conformación de este nuevo calendario se consideró la duración del año en 365,25 días. Dado que cada cuatro años se completaba un día con las fracciones, se decidió el año de 366 días que se llamó bisiesto.

Los 365 días se distribuyen en meses sin tomar en cuenta el mes lunar. Se asignaron 31 y 30 días a los meses en forma alternada. Como no alcanzaban los días para tener seis meses de 31 días, se quitó un día del último mes, febrero, dejándolo con una duración de 29 días, excepto para los años bisiestos que tendría 30. Este calendario, conocido como juliano, entró a regir en el 46 a.C.:

$\begin{array}{llll}\text { 1. } & \text { Martius } & 31 & \text { Marte } \\ \text { 2. } & \text { Aprilis } & 30 & \text { Apolo } \\ \text { 3. } & \text { Maius } & 31 & \text { Júpiter-Maius } \\ \text { 4. } & \text { Junius } & 30 & \text { Juno } \\ \text { 5. } & \text { Quintilis } & 31 & \text { luego Julio } \\ \text { 6. } & \text { Sextilis } & 30 & \text { luego Agosto } \\ \text { 7. } & \text { September } & 31 & \\ \text { 8. } & \text { October } & 30 & \end{array}$

Sobre las fracciones continuas: aplicaciones y curiosidades. Manuel Murillo Derechos Reservados @ 2015 Revista digital Matemática, Educación e Internet (http://tecdigital.tec.ac.cr/revistamatematica/) 


$\begin{array}{rlll}\text { 9. } & \text { November } & 31 & \\ \text { 10. } & \text { December } & 30 & \\ \text { 11. } & \text { Januarius } & 31 & \text { Jano } \\ \text { 12. } & \text { Februarius } & 29-30 & \text { Febro }\end{array}$

Posteriormente, César decretó que el año comenzaría en enero y Quintilis, el mes de su nacimiento, se llamaría ahora julio. Más tarde, Augusto César decretó que el mes Sexto que seguía a julio se llamaría agosto y tendría, al igual que julio, 31 días, día que le quitó a febrero. Al mismo tiempo intercaló la duración de los meses de setiembre en 30 días hasta diciembre en 31, como se le conoce hoy.

Esto último explica por qué setiembre no es el sétimo mes, como sugiere su nombre, al igual que no ocurre con octubre, noviembre y diciembre, cuyos nombres sugieren que son el octavo, noveno y décimo mes, respectivamente.

El año de la reforma se tuvo que alargar hasta 445 días, para que la primavera iniciara el 25 de marzo, como en los tiempos de Numa. Es decir, el 46 a.C. es el más largo antes de Jesucristo.

Con el pasar de los años se logró una mejor precisión en el cálculo de duración del año; consecuentemente, se evidenciaron los defectos del calendario juliano. En 1582, el Papa Gregorio XIII designó al astrónomo italiano Cristóbal Clavio trabajar sobre una reforma del calendario, específicamente en lo referente a los años bisiestos, pues la duración del año no es exactamente 365,25 días, sino más bien 365 días 5 horas 49 minutos y 16 segundos, según las tablas astronómicas elaboradas por la Academia de Toledo en el siglo XIII, por orden expresa de Alfonso X el Sabio (1221-1284), rey de Castilla y de León.

Acorde con las recomendaciones de Clavio, el Papa Gregorio XIII decretó que:

- Será bisiesto aquel año cuya cifra sea divisible por 4, excepto los años seculares, múltiplos de 100, los cuales serán bisiestos únicamente si son divisibles por 400.

- Dado que desde la vigencia del calendario juliano se habían considerado como bisiestos los años que no debieron serlo y ya había un error acumulado de 10 días, se quitarían 10 días al calendario: el día siguiente al 4 de octubre de 1582 (la fiesta de San Francisco de Asís) sería llamado a ser 15 de octubre.

Por lo anterior, 1582 es el año más corto de la cristiandad con 355 días, y los días del 5 al 14 de octubre de ese año no existieron.

De acuerdo con estudios astronómicos, el calendario se adelanta un poco al Sol; cada año gana 26 segundos, lo cual equivale a un día cada 3323 años. Así es probable que se haya perdido un día cuando llegue el año 4000. Por esta pequeña diferencia se ha establecido una regla adicional, cual es que los años múltiplos de 4000 no son bisiestos.

Finalmente, estas reglas que se mencionaron definen al que se conoce como calendario gregoriano; además, algunos sectores de la iglesia ortodoxa se rigen con el calendario juliano para sus celebraciones religiosas.

Actualmente se sabe con mayor precisión que la duración del año es de 365 días, 48 minutos y 46,15 segundos. Los ajustes posibles de los bisiestos para lograr una aproximación a la duración media del año son:

\begin{tabular}{lc} 
Ajuste posible & Error que produce \\
\hline 1 año bisiesto cada 4 años & -11 minutos al año \\
7 años bisiestos cada 29 años & 1 minuto al año \\
8 años bisiestos cada 33 años & -19 segundos al año \\
31 años bisiestos cada 128 años & 1 segundo al año
\end{tabular}


Esta tabla se logra utilizando fracciones continuas. Si se transforma la duración del año en días, esta será $365+\frac{10463}{43200}$ días, observe que:

$$
\frac{10463}{43200}=\frac{1}{4+\frac{1}{7+\frac{1}{1+\frac{1}{3+\frac{1}{5+\frac{1}{64}}}}}}
$$

es decir, $\frac{10463}{43200}=[0 ; 4,7,1,3,5,64]$ y sus primeros cinco convergentes son $c_{1}=0 \mathrm{y}$ :

$$
\begin{aligned}
& c_{2}=\frac{1}{4} \\
& c_{3}=\frac{1}{4+\frac{1}{7}=\frac{7}{29}} \\
& c_{4}=\frac{1}{4+\frac{1}{7+\frac{1}{1}}}=\frac{8}{33} \\
& c_{5}=\frac{1}{4+\frac{1}{7+\frac{1}{1+\frac{1}{3}}}}=\frac{31}{128}
\end{aligned}
$$

Note que con el calendario gregoriano se intercalan 97 años bisiestos cada 400 años, lo cual viene a ser casi igual a 31 años bisiestos cada 128 años, es decir, la distribución que según la tabla anterior, presenta el menor error. De la proporción $\frac{31}{128}=\frac{x}{400}$

resulta $x=96,875$, que es una buena aproximación de los 97 años bisiestos del calendario gregoriano. De esta forma, la manera en que se resolvió el problema de agregar los días en los años bisiestos fue la óptima y se relaciona con la exactitud de las aproximaciones de los convergentes.

Es importante aclarar que estas modificaciones para el nuevo calendario se adoptaron gradualmente por los distintos países, por ejemplo:

$\begin{array}{ll}\text { Año } & \text { Países } \\ 1582 & \text { Italia, España, Portugal, Francia } \\ 1583 & \text { Alemania católica } \\ 1584 & \text { Bohemia, Moravia, Suiza católica } \\ 1586 & \text { Polonia } \\ 1587 & \text { Hungría } \\ 1606 & \text { Siria } \\ 1700 & \text { Dinamarca, Países Bajos } \\ 1701 & \text { Suiza y Alemania protestantes } \\ 1752 & \text { Inglaterra, EUA (colonias inglesas) } \\ 1753 & \text { Suecia } \\ 1873 & \text { Japón } \\ 1914 & \text { Turquía } \\ 1922 & \text { Grecia } \\ 1923 & \text { Rusia }\end{array}$


Así, se adoptó en Rusia hasta 1923, por lo que la conocida Revolución Bolchevique de 1917, llamada la gran revolución de octubre, curiosamente ocurrió en noviembre del nuevo calendario, y en la actualidad se celebra durante dicho mes. Esto se debe a la diferencia de 13 días que hay entre el juliano y el gregoriano: 3 días asociados a los años 1700, 1800 y 1900, luego de la reforma, que no fueron bisiestos en el gregoriano y los 10 días que se eliminaron, del 5 al 14 de octubre de 1582.

Por último, y siempre relacionado con el asunto de corregir las deficiencias que pudiera generar la forma de medir el tiempo, recuerde que la cantidad de segundos que tiene un día es de $60 \cdot 60 \cdot 24=$ 86400 , durante cada día de la semana y durante cada semana del año.

Es sabido que las mareas son el producto de la fuerza de gravitacional que ejerce la Luna sobre la Tierra. Este efecto es mayor sobre la parte de la Tierra que se encuentra enfrente que en el lado opuesto. El resultado es un pequeño alargamiento de la Tierra en dirección de la Luna, que tiene mayor efecto sobre la masa líquida que sobre la parte sólida, lo cual produce las mareas dos veces al día. Las masas de agua producen fricción con las partes bajas del mar, y esta fricción transforma la energía en calor, es decir, la Tierra está perdiendo energía rotacional.

Como lo explica claramente Isaac Asimov en [2], la pérdida del índice de rotación no es fácil de notar; sin embargo, para los astrónomos esto hace que una estrella que se observó en una posición en un tiempo determinado se observe hoy con un corrimiento significativo; lo mismo ocurriría con los eclipses. Cuando la rotación de la Tierra está atrasada en 0,9 segundos se agrega un segundo bisiesto para lograr que el planeta esté sincronizado de nuevo, lo cual es útil y necesario para la navegación, las telecomunicaciones y, como se ha mencionado, para la astronomía.

En 1972 se creó el sistema de resincronización del planeta. En ese momento se agregaron diez segundos para lograr el ajuste, y con ello el año más largo es 1972, que además de ser bisiesto, tiene diez segundos más.

Desde 1972 hasta hoy se han añadido en total 34 de estos segundos, siempre a finales de junio o de diciembre, el antepenúltimo de ellos en 2005, el penúltimo el 31 de diciembre del 2008 y el último el 30 de junio del 2012.

\section{Bibliografía}

[1] Andrews, G. Number Theory. Dover Publication Inc, 1971.

[2] Asimov, I. Fronteras. España: Ediciones B, S.A., 1991.

[3] Beskin, N. Fracciones maravillosas. Moscú: Editorial Mir, 1987.

[4] Gauss, K. Disquisitiones Arithmeticae, 1801. (Traducido al español por Ángel Ruiz y otros). Colombia: Academia Colombiana de Ciencias Exactas, Físicas y Naturales, 1995.

[5] Jones, B. Teoría de los Números. México: Edit. Trillas, 1969.

[6] Murillo, M.; González, F.Teoría de los números. Editorial Tecnológica de Costa Rica, 2012.

[7] Niven, I.; Zuckerman, H. Introducción a la Teoría de los Números. México: Editorial Limusa, 1976.

[8] Pettofrezzo, A. \& Byrkit, D. Introducción a la Teoría de los Números. España: Editorial Prentice Hall, 1972.

[9] Ruiz, A. Historia y Filosofía de las Matemáticas. Costa Rica: Editorial de la Universidad Estatal a Distancia, 2003.

[10] Vorobiov, N. Criterios de divisibilidad. Lecciones populares de matemática. Moscú: Editorial Mir, 1984.

Sobre las fracciones continuas: aplicaciones y curiosidades. Manuel Murillo

Derechos Reservados @ 2015 Revista digital Matemática, Educación e Internet (http://tecdigital.tec.ac.cr/revistamatematica/) 